\title{
Matrix Product States for dynamical simulation of infinite chains
}

\author{
M. C. Bañuls, ${ }^{1, *}$ M. B. Hastings, ${ }^{2}$ F. Verstraete, ${ }^{3}$ and J. I. Cirac ${ }^{1}$ \\ ${ }^{1}$ Max-Planck-Institut für Quantenoptik, Hans-Kopfermann-Str. 1, 85748 Garching, Germany. \\ ${ }^{2}$ Microsoft Research, Station Q, CNSI Building, University of California, Santa Barbara, CA, 93106. \\ ${ }^{3}$ Fakultät für Physik, Universität Wien, Boltzmanngasse 5, A-1090 Wien, Austria.
}

(Dated: May 29, 2018)

\begin{abstract}
We propose a new method for computing the ground state properties and the time evolution of infinite chains based on a transverse contraction of the tensor network. The method does not require finite size extrapolation and avoids explicit truncation of the bond dimension along the evolution. By folding the network in the time direction prior to contraction, time dependent expectation values and dynamic correlation functions can be computed after much longer evolution time than with any previous method. Moreover, the algorithm we propose can be used for the study of some non-invariant infinite chains, including impurity models.
\end{abstract}

PACS numbers: 03.67.Mn, 02.70.-c, 75.10.Jm

Numerical simulation has become a fundamental tool to study quantum many-body systems in condensed matter physics. Unfortunately, the exponential scaling of the dimension of the Hilbert space with system size means that brute-force methods are only practical for very small system sizes. However, other techniques based on Matrix Product States (MPS) [1, 2, 3, 4, 5], such as DMRG [6], achieve excellent results in one dimension.

The success of DMRG methods is based on the fact that many interesting physical states, including the ground states of many local Hamiltonians, can be well approximated by a MPS. For a chain of $N d$-dimensional systems, this has the form

$$
|\Psi\rangle=\sum_{i_{1}, \ldots i_{N}=1}^{d} \operatorname{tr}\left(A_{1}^{i_{1}} \ldots A_{N}^{i_{N}}\right)\left|i_{1}, \ldots i_{N}\right\rangle .
$$

Each $A_{k}^{i}$ is a $D$-dimensional matrix. An important feature of MPS is that expectation values of local operators can be efficiently computed, allowing them to be used variationally. DMRG excels in the computation of static properties of finite chains with local Hamiltonians, where the required bond dimension grows slowly with the size of the system 7, 8], and of translationally invariant infinite chains $[9,10]$. However, these methods can break down in time-dependent problems far from equilibrium and also encounter difficulties in dealing with infinite chains containing impurities.

MPS algorithms for non-equilibrium dynamics 11, 12, 13, 14, 15] work well when the system is close to its ground state, but when the system is far from equilibrium the entanglement entropy may grow linearly in time and the dimension $D$ required to describe the system will grow exponentially [16, 17, 18], causing these methods to break down. Although improved algorithms have been developed based on finite propagation speed of correlations [19], all known methods are limited to special cases or short times.

Here we propose an alternative method to compute

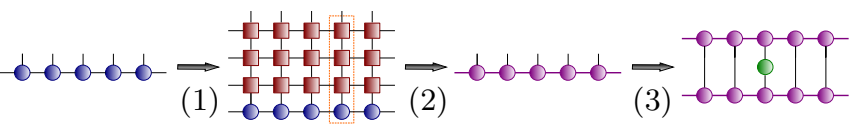

FIG. 1: Standard time evolution with MPS. We start with a MPS state, represented here by a chain of circles (tensors) connected by summed indices, with open lines for the physical spin indices. On this state, a sequence of MPO is applied for each step of evolution (1), and the result is truncated (2) to the maximal bond dimension $D$. After iterating the evolution, expectation values are computed (3) in the final state.

both ground states and dynamical quantities for infinite chains within the MPS formalism. Based on the transverse contraction of the tensor network, it allows the study of problems not accessible by other methods, such as an impurity in an infinite system. It enables the calculation of time-dependent expectation values of local observables and of few body correlation functions at different times, in a new much simpler way. Moreover, by a folding of the network described below, the new method enables dynamical studies that range much further in time than any other existing method.

The standard way of computing dynamical quantities with the MPS formalism starts with a state that is (exactly) described by a MPS (11). Then, some evolution operator is applied to it for a given time, making use of a Suzuki-Trotter expansion [20] of the total evolution operator. Within each discrete time step, the evolution operator is broken down into a product of operators. In particular, for a nearest neighbor Hamiltonian $H=\sum_{i} h_{i, i+1}$, we may write $e^{-i H \delta} \approx e^{-i H_{e} \delta / 2} e^{-i H_{o} \delta} e^{-i H_{e} \delta / 2}$, where $H_{e}$ $\left(H_{o}\right)$ contains the $h_{i, i+1}$ terms with even (odd) $i$, so that each exponential factor is a product of mutually commuting local terms. Alternatively, the evolution operator can be decomposed as a product of translationally invariant Matrix Product Operators (MPO) 21]. The action of one step of evolution on the MPS can be computed by applying the corresponding sequence of operators (Fig. 1) 


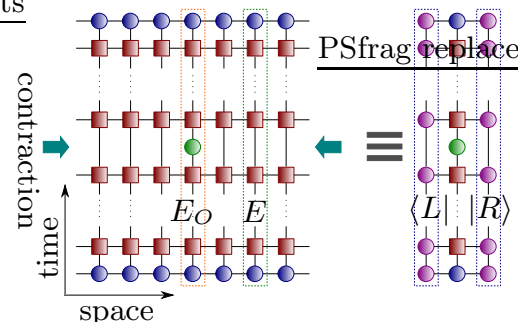

(a)Transverse contraction along space direction renders a finite $2 \mathrm{D}$ network.

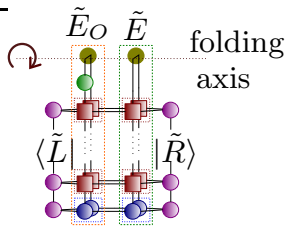

(b)Transverse contraction of folded network.

FIG. 2: Expectation value $\langle O(t)\rangle$ with the basic transverse method (a) and with folding (b), where operators for the same time step are grouped together in a double effective operator.

to yield a MPS with larger bond dimension. This must be truncated to keep the best MPS description of the evolved state with fixed dimension, $D$. After repeating this procedure for the required number of steps, expectation values can be calculated in the evolved state. The accuracy of the description will however drop exponentially with the successive truncations.

Our new method avoids this explicit truncation on the bond dimension of the evolved MPS. The basic idea is to look at the quantity that we want to compute, say the time dependent expectation value of some local operator, $\langle\Psi(t)|O| \Psi(t)\rangle$, as the contraction of a two dimensional tensor network, and perform it, not along time, but in the direction of space (see Fig. 2(a) . To construct the network, we start from the initial MPS and, for every evolution step, apply the proper MPOs. Repeating this for the required number of evolution steps, we construct the exact evolved MPS (within the Trotter approximation), as no truncation is carried out. Finally, we apply the local operator $O$ and contract with the Hermitian conjugate of the evolved state as constructed before.

The procedure above produces a two dimensional network, infinite in the spatial direction as the original MPS, but finite along the time direction. The expectation value we want to compute can now be written as [5],

$$
\langle\Psi(t)|O| \Psi(t)\rangle=\lim _{k \rightarrow \infty} \operatorname{tr}\left(E_{\ell}^{[-k]} \ldots E^{[-1]} E_{O}^{[0]} E^{[1]} \ldots E_{r}^{[k]}\right),
$$

where $E(t)=\sum_{i} \bar{A}^{i}(t) \otimes A^{i}(t)$ is the transfer matrix of the evolved state, $E_{O}(t)=\sum_{i, j}\left[\bar{A}^{i}(t) \otimes A^{j}(t)\right]\langle i|O| j\rangle$ contains the only application of the single-body operator, and the bracketed superindices on each transfer matrix indicate the site of the chain. For a translationally invariant MPO representation of the evolution operator, the network retains the invariance [27] and the transfer matrix is the same on every site, except for the single one on which $O$ acts 28]. If the largest eigenvalue of $E(t), \lambda$, is non-degenerate, $E^{k}(t) \underset{k \rightarrow \infty}{\longrightarrow} \lambda^{k}|R\rangle\langle L|$. Effectively, we may then substitute the left and right semi-infinite lattices at both sides of the operator by the left and right eigenvectors of $E(t)$ corresponding to the largest eigen-

value, $\langle L|$ and $|R\rangle$,

$$
\langle O(t)\rangle=\frac{\langle\Psi(t)|O| \Psi(t)\rangle}{\langle\Psi(t) \mid \Psi(t)\rangle}=\frac{\left\langle L\left|E_{O}\right| R\right\rangle}{\langle L|E| R\rangle} .
$$

We now specify the algorithm for computing timedependent expectation values in translationally invariant infinite chains. The first step is to find the best MPS approximation, with given bond dimension $D$, to the dominant eigenvectors of $E(t)$. To this end, we repeatedly apply the transfer matrix $E(t)$ (already written as a MPO along the time direction, see Fig. 2(a) to the left and to the right of an arbitrary initial MPS vector and truncate the result to the chosen $D$, using the technique for two dimensional tensor networks introduced in [14, 22], until convergence is achieved. The procedure yields a MPS approximation to the eigenvectors, with the truncation taking always place in the space of transverse vectors. The second step, computing the numerator and the denominator in (2), can be done very efficiently, as each term is a contraction of a MPO acting between a pair of MPS. The adaptation of the method to the case of imaginary time evolution is straightforward, so that it is also useful for finding ground state properties. In this case, the eigenvector calculation is similar to that in transfer matrix DMRG algorithms for thermal states [23].

With this approach, we study an infinite chain with an impurity. We consider an Ising chain,

$$
H=-\left(\sum_{i} \sigma_{z}^{i} \sigma_{z}^{i+1}+g_{i} \sigma_{x}^{i}\right),
$$

with $g_{i}=1 \forall i \neq 0$, and the impurity represented by a different value of the field at site $i=0, g_{0}$. The system is started in a product MPS and imaginary time evolution is applied for a long time, so that we approach the ground state. Then we compute the site dependent magnetization, $\left\langle\sigma_{x}^{[i]}\right\rangle$. Such a calculation cannot be easily done with a purely invariant method as iTEBD [10], because the presence of a singular site will affect a cone of tensors as time increases. However, with the transverse method, the computation of $\langle L|$ and $|R\rangle$ is not modified by the presence of the impurity [24]. Thus the cost of computing the expectation value of a local operator acting on the position $i=0$ in the ground state of this chain will be the same as in the translationally invariant case, while applying the operator at $i \neq 0$ will reduce to the contraction of a $2 \mathrm{D}$ tensor network of width $i+3$ (Fig. (3).

The capabilities of the transverse method regarding real time evolution can be further illustrated by the computation of two-body correlators at different times. If we consider two different times, $t_{2}>t_{1}$, we may write

$$
\begin{aligned}
\langle\Psi(0) & \left.\left|O_{2}^{[x]}\left(t_{2}\right) O_{1}^{[x+\Delta]}\left(t_{1}\right)\right| \Psi(0)\right\rangle \\
= & \left\langle\Psi(0)\left|U\left(t_{2}, 0\right)^{\dagger} O_{2}^{[x]} U\left(t_{2}, t_{1}\right) O_{1}^{[x+\Delta]} U\left(t_{1}, 0\right)\right| \Psi(0)\right\rangle \\
= & \frac{\left\langle L\left|E_{O_{2}\left(t_{2}\right)} E^{\Delta-1} E_{O_{1}\left(t_{1}\right)}\right| R\right\rangle}{\left\langle L\left|E^{\Delta+1}\right| R\right\rangle}
\end{aligned}
$$




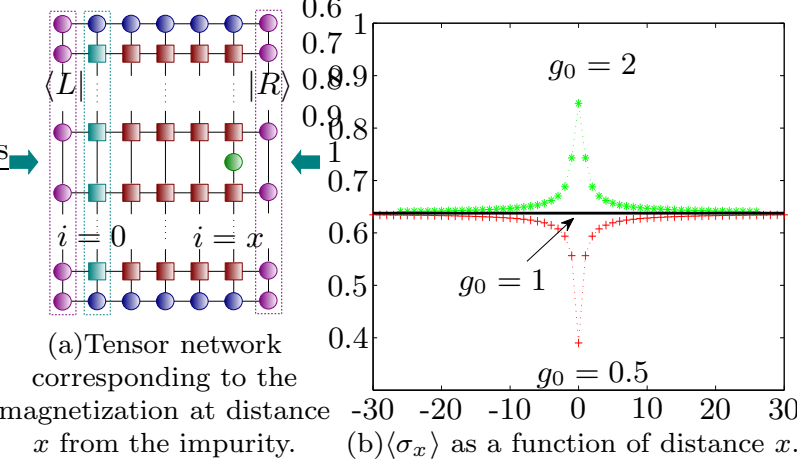

FIG. 3: Ising chain with magnetic impurity at the origin.

where $E$ is the transfer matrix resulting from evolution until time $t_{2}$, and $E_{O_{i}(t)}$ are the corresponding MPO containing the action of each single-body operator [25].

In particular, if both operators act on the same site $(\Delta=0)$, the computation has the same cost as one single expectation value. If $\Delta \neq 0$, computing (44) requires instead the contraction of a two dimensional network of width $\Delta+3$. This is done by applying one MPO at a time and truncating to the closest MPS with the given bond $D$. Since the network is now finite in both directions, this last phase of the contraction can be done either in the spatial or in the time direction.

The success of the transverse approach will depend on whether the transfer matrix of the evolved MPS has a non-degenerate dominant eigenvector which can be approximated by a MPS of reduced dimension. Our implementation shows that the procedure achieves comparable results to the standard contraction [10] in a translationally invariant chain. The transverse method offers the advantage of being applicable to dynamical situations in which translational symmetry is broken by a small number of sites, such as a chain with impurities, or a semiinfinite system, but it is also limited to short times.

However, there is a more efficient representation of the entanglement in the transverse eigenvectors. In the MPO representing the transfer matrix of the evolved MPS, tensors that lie at the same distance from the center (occupied by the physical operator $O$ as in Fig. 2(a) correspond to the same time step, coming from a certain term and its adjoint in the Trotter decomposition. We can group such pairs together in a new MPO by "folding" the original MPO (see Fig. 2(b)]. The folding operation can be understood as performing the equivalent asymmetric contraction $\langle\Psi(t)|O| \Psi(t)\rangle=\langle\Phi|(O|\Psi(t)\rangle \otimes|\bar{\Psi}(t)\rangle)$ where $|\bar{\Psi}(t)\rangle$ is the complex conjugate of the evolved vector and $|\Phi\rangle=\otimes_{k} \sum_{i_{k}=1}^{d}\left|i_{k} \bar{i}_{k}\right\rangle$ is the product of (unnormalized) maximally entangled pairs between each site of the chain and its conjugate. In our scheme, the ket is now the tensor product of two tensor networks corresponding to $|\Psi(t)\rangle$ and its conjugate. We may then group together each tensor in $|\Psi\rangle$ with the corresponding one in $|\bar{\Psi}\rangle$, and define an effective tensor network of higher bond dimension and physical dimension $d^{2}$, which can now be contracted using again the transverse technique.

This folded transverse method allows us to explore the dynamics until much longer times than any other procedure. We may get some physical intuition for this improvement by looking at a single localized excitation that propagates freely with velocity $v$. After time $t$, sites $x \pm v t$ in the evolved state become entangled. If we look instead at the transverse MPS obtained contracting the network from the right until $x+v t$, it is easy to see that all time sites are in a product, except for those corresponding to the instant $t$. These sites occupy symmetric positions around the center of the network, so that folding groups them together in a single site which will be in a product state with all the rest.

As a first benchmark for the new method, we simulate the dynamics of states far from equilibrium under the Ising Hamiltonian (3) with uniform magnetic field $g$. The initial state $\left|\Psi_{0}\right\rangle=\otimes_{i} \frac{1}{\sqrt{2}}\left(|0\rangle_{i}+|1\rangle_{i}\right)$ is evolved with a constant Hamiltonian and the results of the transverse method with and without folding are compared to the exact results (Fig. 4). For very short times the Trotter error dominates in both methods. However, while for the transverse procedure (as for iTEBD) truncation error becomes soon dominant, and the results deviate abruptly from the exact solution, the accuracy of the folded version is maintained for much longer times.

To test the method on a more general problem, we repeat the test for a non-integrable Hamiltonian, $H=$ $-\left(\sum_{i} \sigma_{z}^{i} \sigma_{z}^{i+1}+g \sigma_{x}^{i}+h \sigma_{z}^{i}\right)$. For this case there are no exact results, but we may compare the folded computation to the iTEBD simulations with a similar Trotter error (Fig. (4). Again we check that the accuracy of the folded procedure for comparable bond dimension reaches much longer times. Moreover, remarkably enough, even when the results from the folded method start deviating (from those to which iTEBD converges for large $D$ ), they do so in a smooth way, so that, in contrast to other procedures, they continue to qualitatively describe the evolution for long times.

This can be seen in a more precise way by looking at the truncation error. At a certain time, this can be estimated by looking at the error in the right eigenvector for a given bond dimension with respect to the best eigenvector obtained, i.e. that for the highest $D$. If we plot (Fig. (4) the bond dimension required to achieve a fixed truncation error, we observe that, although the $D$ required for a high precision grows exponentially with time, with a relatively low bond $D<100$ a qualitative description of the dynamics is reproduced, that lies within $1 \%$ of the exact solution well beyond times $t>10$.

From the discussion above the transverse method, combined with the folding technique, represents a very promising tool for the dynamical studies of one dimen- 

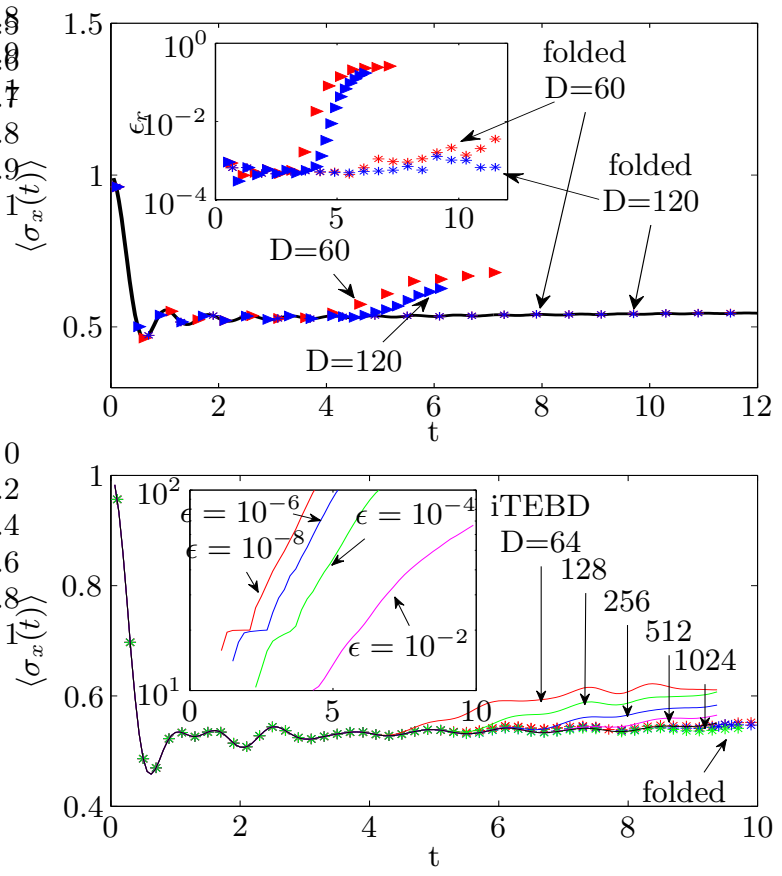

FIG. 4: Magnetization as a function of time. For the Ising model (up) results for the transverse method (triangles) are compared to the folded version (stars) for $D=60,120$. The relative error with respect to the exact result (solid line) is shown in the inset. For the non-integrable model (down), results with the folded approach for $\mathrm{D}=60$ (red), 120 (blue), 240 (green) are compared to those of iTEBD (solid lines) for increasing values of $D$. In the inset, the required value of $D$ as a function of time, for different levels of accuracy.

sional systems. The first results show the applicability of the method even to non-integrable systems, allowing the simulation of longer evolution times than any other technique, and a qualitative description of the dynamics until even later. This opens the door for the study of physical problems not accessible until now for numerical methods, including the dynamics of phase transitions, out-of-equilibrium states and thermalization problems. The present formalism might also prove very valuable in the context of extracting spectral information for quantum impurity problems, the central problem in dynamical mean field theory. The big advantage of our method is that we can deal with real frequencies, and no analytic continuation from imaginary frequencies is needed as in the case of Monte Carlo simulations. In contrast, the main limitation would be its exclusive applicability to one dimensional systems. Finally, although the method has been described for infinite chains, it is easy to adapt the technique for the dynamical study of finite systems.

Note: An independent derivation in [26], in the context of concatenated tensor network states, lead to a similar network to describe time-evolved states.

We acknowledge D. Pérez-García and J. J. GarcíaRipoll for discussions and T. Nishino for pointing out Ref. [24]. This work was supported by DFG through Ex- cellence Cluster MAP and FOR 635, and by EU project SCALA.

* Electronic address: banulsm@mpq.mpg.de

[1] I. Affleck, T. Kennedy, E. H. Lieb, and H. Tasaki, Commun. Math. Phys. 115, 477 (1988).

[2] A. Klumper, A. Schadschneider, and J. Zittartz, J. Phys. A 24, L955 (1991); Z. Phys. B 87, 281 (1992).

[3] M. Fannes, B. Nachtergaele, and R. F. Werner, Commun. Math. Phys. 144, 443 (1992).

[4] F. Verstraete, D. Porras, and J. I. Cirac, Phys. Rev. Lett. 93, 227205 (2004).

[5] D. Perez-García, F. Verstraete, M. M. Wolf, and J. I. Cirac, Quantum Inf. Comput. 7, 401 (2007).

[6] S. R. White, Phys. Rev. Lett. 69, 2863 (1992); U. Schollwöck, Rev. Mod. Phys. 77, 259 (2005).

[7] F. Verstraete and J. I. Cirac, Phys. Rev. B 73, 094423 (2006).

[8] M. B. Hastings, J. Stat. Mech. 2007, P08024 (2007).

[9] S. Östlund and S. Rommer, Phys. Rev. Lett. 75, 3537 (1995).

[10] G. Vidal, Phys. Rev. Lett. 98, 070201 (2007).

[11] M. A. Cazalilla and J. B. Marston, Phys. Rev. Lett. 88, 256403 (2002).

[12] S. R. White and A. E. Feiguin, Phys. Rev. Lett. 93, 076401 (2004).

[13] A. J. Daley, C. Kollath, U. Schollwock, and G. Vidal, J. Stat. Mech. 2004, P04005 (2004).

[14] F. Verstraete, J. J. García-Ripoll, and J. I. Cirac, Phys. Rev. Lett. 93, 207204 (2004).

[15] G. Vidal, Phys. Rev. Lett. 91, 147902 (2003).

[16] P. Calabrese and J. Cardy, J. Stat. Mech. 2005, P04010 (2005).

[17] N. Schuch, M. M. Wolf, F. Verstraete, and J. I. Cirac, Phys. Rev. Lett. 100, 030504 (2008).

[18] T. J. Osborne, Phys. Rev. Lett. 97, 157202 (2006).

[19] M. B. Hastings, Phys. Rev. B 77, 144302 (2008).

[20] H. F. Trotter, Proc. Amer. Math. Soc. 10, 545 (1959); M. Suzuki, Phys. Lett. A 146, 319 (1990).

[21] V. Murg, J. I. Cirac, B. Pirvu, and F. Verstraete, arXiv:0804.3976 [quant-ph].

[22] V. Murg, F. Verstraete, and J. I. Cirac, Phys. Rev. A 75, 033605 (2007).

[23] R. J. Bursill, T. Xiang, and G. A. Gehring, J. Phys. Condens. Matter 8, L583 (1996); X. Wang and T. Xiang, Phys. Rev. B 56, 5061 (1997).

[24] S. Rommer and S. Eggert, Phys. Rev. B 59, 6301 (1999).

[25] F. Naef, X. Wang, X. Zotos, and W. von der Linden, Phys. Rev. B 60, 359 (1999).

[26] R. Hübener, V. Nebendahl, and W. Dür, arXiv:0904.1925v1 [quant-ph].

[27] Using the standard decomposition of the evolution operator into commuting products, the resulting network has translational symmetry with period two, so that the argument can be easily adapted substituting $E$ for the product of two contiguous transfer matrices, $E_{e} E_{o}$.

[28] In the infinite limit we do not need to consider the vector terms at the edges, $E_{\ell}^{[-k]}$ and $E_{r}^{[k]}$, which would also be different for the finite case. 